PharmacoEconomics \& Outcomes News 865, p2 - 31 Oct 2020

\title{
Women disadvantaged by employment-based health financing
}

Employment-based health financing schemes "are likely to favour men and can undermine progress towards gender equitable universal health coverage", according to a BMJ article on women's health and gender inequalities.

Despite global commitments repeatedly being made to improve women's access to healthcare, the authors note that "women remain disproportionately underserved, and their basic health needs remain unmet" and believe that barriers to healthcare are exacerbated by lower income, higher burden of unpaid care work and limited decision-making power over resources often experienced by women. In this article, Vijayasingham et al discuss how health financing and entitlement systems linked to employment can further disadvantage women in equal access to healthcare.

Employment-based health financing schemes may be mandatory, semi-mandatory or voluntary and typically consist of employees, employers and/or the state contributing towards a pooled fund for the provision of certain health entitlements for the contributing individuals and possibly their dependents. Criticisms of employment-based health financing include the implication that healthcare is a benefit of employment, rather than a human right, as well as the undermining of universal health coverage by linking employment status and contributions to healthcare entitlements. Furthermore, the authors state that the system is "unstable, fragmented, and inequitable" when utilised as a source of health revenue, particularly during an economic crisis such as the COVID-19 pandemic which has seen approximately 47.5 million people in the US lose access to employment-linked healthcare due to job losses.

Gender inequalities in employment terms between men and women, as well as unequal burdens of unpaid and domestic work, contribute to the issue and "are likely to be exacerbated in the future". The informal sector, which employs up to $75 \%$ of employed women in Latin America and up to $90 \%$ in Africa and South Asia, generally offers occupations with lower pay, longer hours, and no social protection. Non-standard employment arrangements, which are increasing in the formal sector and often depicted as a flexible work option for women to balance employment and domestic responsibilities, frame workers as self-employed and therefore lack job security and health-related social protection.

Women are also more likely to have fragmented work lives due to reproduction, unemployment (voluntary and involuntary) and care responsibilities, which exposes them to discontinuity in health coverage via employment-based schemes. Additionally, lower value is placed on unpaid versus paid work in employment-based models, which reduces healthcare entitlements and coverage.

Vijayasingham et al believe that the "path towards achieving universal health coverage must include gender and other equity considerations beyond socioeconomic position and income", and that an individual's access to healthcare should be unaffected by employment status, life roles or income. There is a need for "safety nets" to be provided to allow continued, equal coverage in countries which already have established employment-based health financing, whilst systems-level reform should also be prioritised to encourage "mandatory, universal, and primarily tax-based financing approaches that separate entitlement to health benefits from contribution". Caregiving and childbearing should be valued as equivalent to paid employment, with regular, affordable premiums and parallel non-contributory schemes ensuring access to equitable care, as well as consideration of people employed through non-standard arrangements. "The gendered effect of the covid-19 crisis, including its effect on unequal care burdens and employment, could be the external shock and trigger needed to produce a change and the introduction of wide reaching reforms to deal with the gendered inequities of healthcare access across lifetimes", concluded the authors. 